\title{
In situ dynamic X-ray micro-CT for additive manufactured parts
}

Jan Dewanckele ${ }^{1}$, Frederik Coppens ${ }^{1}$, Wesley De Boever ${ }^{2}$, Marijn Boone ${ }^{3}$ and Luke Hunter ${ }^{4}$

${ }^{1}$ TESCAN, Belgium, ${ }^{2}$ Tescan, Belgium, ${ }^{3}$ TESCAN XRE, Belgium, ${ }^{4}$ Tescan, United States

The evolution of additive manufacturing (AM) over the last decade has been impressive and looks to be revolutionary as a disruptive technique in the overall manufacturing arena. However, there are still a number of obstacles to overcome concerning the quality of the final part. X-ray micro-CT is a non-destructive technique used to create highly detailed 3D volumes of intact samples and is widely used throughout an AM part's lifecycle; process development, process monitoring, and final part quality. On the other hand, dynamic micro-CT enables the non-destructive inspection of processes inside a sample under altering external conditions (such as load or temperature). Dynamic CT, in particular, refers to the most advanced sub-set of time-resolved X-ray imaging, where a sample is imaged continuously as it is changing. The difference between dynamic and time-lapse can be thought of as the difference between a smooth motion picture and a stop motion animation. The advantage of performing continuous acquisition (dynamic CT) lies in the ability to perform uninterrupted, real in situ experiments. A material undergoing change cannot always be predicted, and by utilizing dynamic CT we can now more accurately understand these processes.

The great advantage of using dynamic CT during compression of additive manufactured samples is that no crucial deformation information is lost, because no pauses are installed during the acquisition. Moreover, the relaxation of the sample becomes irrelevant as the sample is continuously compressed. By tuning the scan (rotation) speed and compression speed of the Deben stage, it becomes possible to reconstruct specific time windows afterwards. These 'sliding time windows' can be arbitrarily set to fully characterize the deformation inside the material afterwards.

In this work, we demonstrate continuous acquisitions for dynamic loading experiments of plastic 3D printed parts. The possibilities and limitations of this new approach in dynamic in-situ imaging will be discussed. Six different parts were compressed using a Deben stage in a TESCAN CoreTOM micro-CT system. The behavior of the infill patterns was evaluated during the complete compression experiment. In total, for each sample, 220 individual reconstructions were acquired with a spatial resolution of $59 \mu \mathrm{m}$ voxel size and a temporal resolution of 5.8 seconds. 

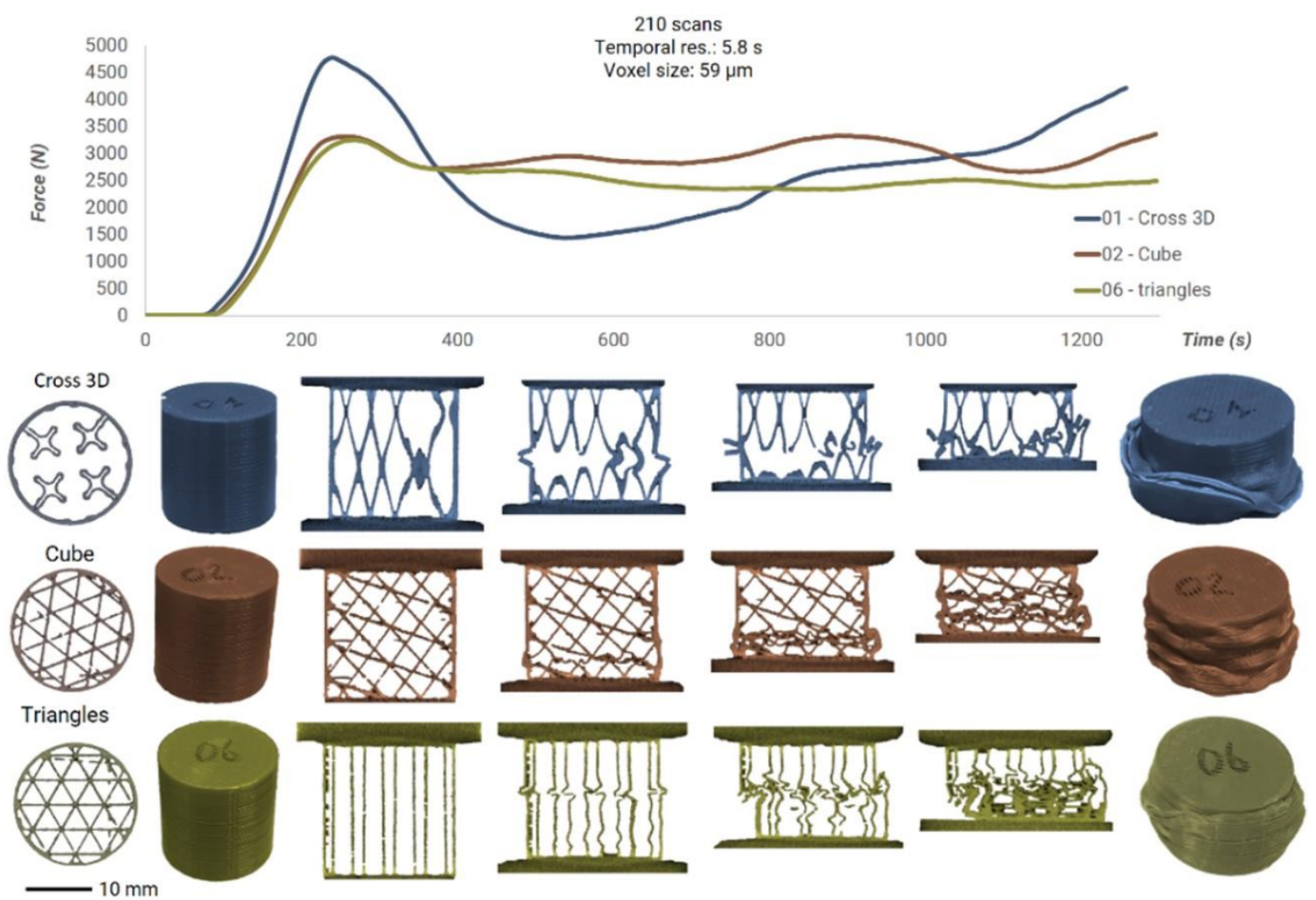

Figure 1. Dynamic X-ray micro-CT experiment to evaluate the infill pattern of different 3D printed parts. The samples were compressed up to $5 \mathrm{kN}$. 